\title{
Die fünf Ziele des Elterngelds im Spannungsfeld von Politik, Medien und Wissenschaft
}

\author{
The five goals of the new German parental leave benefit in the focus of \\ politics, media and science
}

\begin{abstract}
Zusammenfassung:
Der Diskurs über das 2007 eingeführte Elterngeld und dessen politische Legitimation hängen eng mit der Wirkung auf bestimmte Ziele zusammen. Welche primären Ziele das Elterngeld hat, wird in den Sphären Politik, Medien und Wissenschaft unterschiedlich beantwortet. Der Beitrag zeigt die jeweils charakteristischen Merkmale der Elterngeld-Diskurse in diesen Bereichen und durch welche Mechanismen diese wechselseitig verknüpft sind. Empirische Grundlage ist eine Frequenzund Valenzanalyse von Zeitungsartikeln für 2004 bis 2012, die mit einer Inhaltsanalyse parlamentarischer Dokumente kombiniert wird. Die Analysen belegen, dass das Elterngeld in allen drei Sphären fünf Zieldimensionen hat. Sie verdeutlichen, dass die Definition von Zielen des Elterngelds der Schlüssel zum Verständnis der Diskurse und der Bewertung des Bundeselterngeld- und Elternzeitgesetzes (BEEG) in der Öffentlichkeit ist. Basierend auf Regressionsanalysen sind der Anlass der Berichterstattung und die Summe der attribuierten Ziele zentrale Faktoren für die Valenz von Zeitungsartikeln. Demnach ist für die Politik eine Kommunikationsstrategie vielversprechend, die den Fünfklang an Zielen betont.
\end{abstract}

Schlagwörter: Elterngeld, Elternzeit, Familienpolitik, Familie, Diskursanalyse, Medien, komplementäre Zielstruktur, Fertilität, Gleichstellung

\begin{abstract}
:
In Germany, the discourse about the incomerelated parental leave policy introduced in 2007 and its legitimisation strongly depends on its effects in the context of certain goals. However, the question of which goals the parental leave policy actually has, is answered controversially in the arenas of politics, media and science. This article shows the characteristics of the different discourses about the parental leave policy in these arenas and the mechanisms behind their interaction. Empirically, frequency analyses and valency analyses of newspapers between 2004 and 2012 are carried out which are combined with content analyses of documents of the German Federal Parliament. The analyses confirm that the different goals can be categorised into five groups. The results show that the definition of goals is the key for understanding the discourses and the public judgement of the parental leave policy. OLS regressions show that the main factors for a positive or negative disposition in the media are the initial reason for the article and the number of considered goals. Hence, for politicians, a communication strategy stressing all five goals is promising.
\end{abstract}

Key words: parental leave benefit, paternity leave, family policy, family, discourse analysis, media, complementary goals, fertility, gender equality 


\section{Einleitung}

Als der Deutsche Bundestag am 29. September 2006 das Bundeselterngeld- und Elternzeitgesetz (BEEG) verabschiedete, hat er einen Paradigmenwechsel in der deutschen Familienpolitik eingeleitet. Dem entsprechend wurde das Elterngeld in der Politik, in den Medien und in der Wissenschaft sehr intensiv begleitet. Gerade aufgrund dieses Paradigmenwechsels bedarf dieses neue Instrument einer substanziellen Outcome-Legitimation, die hier im Easton'schen Sinne eines zyklischen Verständnisses von Politikprozessen verstanden wird (Easton 1965). Das Elterngeld muss Erfolge aufweisen, um angesichts inhaltlicher Kritikpunkte und Haushaltskonkurrenz gegenüber anderen Maßnahmen dauerhaft bestehen zu können. Dies ist bei einer inkrementalistischen Veränderung eines etablierten Instruments - wie beispielsweise bei einer Kindergelderhöhung - nicht in dieser Intensität notwendig wie bei dem neuen Instrument Elterngeld. Folgt man der Terminologie des Policy Cycles (Jann/Wegrich 2003), ist die Evaluierung maßgeblich für den weiteren Politikprozess, wobei Evaluierung sowohl wissenschaftliche Analysen als auch die öffentliche Debatte über Ziele und Erfolge einschließt (ibd.: 93).

Für diese Legitimationsfrage - aber auch für den Entstehungsprozess des BEEG ist der Diskurs in Medien, Politik und Wissenschaft essentiell. Zentral ist dabei die Definition und Erwartung von Zielen des Elterngelds. Alle drei Sphären definieren Ziele des Elterngelds und auch ihre Hierarchie. Die Wissenschaft analysiert mögliche Wirkungen; politische Akteure, aber auch Medien, nutzen wissenschaftliche Befunde und amtliche Daten, um das Elterngeld anhand der Ziele zu legitimieren oder aber zu delegitimieren.

Dieser Beitrag analysiert diese Diskurse und ihre Interdependenzen, um Einflussmechanismen auf die öffentliche Bewertung und damit auf die Outcome-Legitimation des Elterngelds zu beleuchten. Ein besonderer Fokus wird auf die Definition von Zielen gelegt, da die Bewertung des Elterngelds erheblich von der Auswahl der Ziele, an denen es gemessen wird, abhängt. Beispielsweise hat ein Befund über (ausbleibende) Effekte auf die Geburtenrate nur dann eine Relevanz für die Bewertung des Elterngelds, wenn dies als wichtiges Ziel gesehen wird. Bezüglich der Zieldefinition wird auch versucht, systematische Widersprüche zwischen Politik und Medien aufzuzeigen.

Diese Analyse versteht sich auch als Grundlage für Beiträge, bei denen die Wirkungen des Elterngelds auf bestimmte Ziele analysiert werden (z.B. Geyer et al.; Trappe; Bujard/Passet in diesem Band). Die Analyse von Diskursen und Zieldefinitionen versucht, die einzelnen Wirkungsstudien mit den Analysen zu Legitimation und Politikprozess zu verbinden.

Es gibt keinen offiziellen Zielkanon des BEEG. Da die Auswahl der Ziele ein Schlüssel zur Bewertung des Elterngelds, also seiner Legitimation und letztendlich damit für die Dauerhaftigkeit dieser Policy ist, versucht der Beitrag, die Ziele des Elterngelds auf empirischer Basis zu prüfen. Von den grundsätzlichen Zieldimensionen der Familienpolitik (vgl. Bujard 2011: 372; Gerlach 2004: 113ff.; Kaufmann 2002: 426ff.; Wingen 1997: 50ff.) sind außer Bildung und Gesundheit alle weiteren Ziele potenziell vom Elterngeld beeinflussbar: Einkommen, Zeit, Gleichstellung, Arbeitsmarktpartizipation und Fertilität. Die Präsenz dieser fünf Ziele wird getrennt für politische, wissenschaftliche und mediale Diskurse anhand von parlamentarischen Dokumenten und einer Medienanalyse vorgenommen. 
Empirischer Kern ist eine Frequenz- und Valenzanalyse von Zeitungsartikeln zum Elterngeld zwischen 2004 und 2012, bei der Anlässe, berücksichtigte Ziele, Timing und Bewertungen im Zeitverlauf dargestellt und mit multivariaten Regressionen analysiert werden. Dabei dient die Systematisierung der Ziele auf Basis des Fünfklangs der Vergleichbarkeit, um im zweiten Schritt die Zielhierarchien zwischen Gesetzgebungsverfahren und Medien $^{1}$ zu vergleichen.

Abbildung 1: Kontexte des Elterngelds

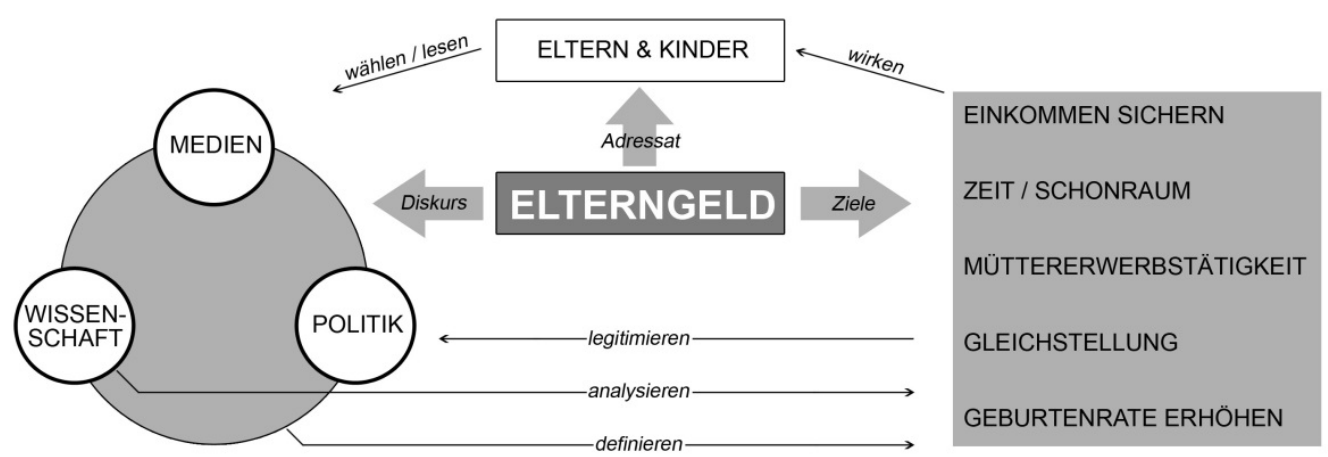

Quelle: Eigene Darstellung.

Abbildung 1 skizziert den kontextuellen Rahmen dieser Analyse zum Elterngeld, speziell die Interaktion von Adressaten, Diskursen und Zielen. Demnach ist der öffentliche Diskurs - der anhand der Medienanalyse empirisch nachgezeichnet wird - durch eine Interaktion zwischen den Sphären Medien, Wissenschaft und Politik gekennzeichnet, wobei die fünf Ziele den zentralen inhaltlichen Bezugspunkt des Diskurses darstellen.

Zum Aufbau dieses Artikels: In Kapitel 2 werden die Diskurse innerhalb der drei Sphären Politik, Medien und Wissenschaft sowie die wechselseitigen Beeinflussungen analysiert mit besonderem Schwerpunkt auf der Entstehung des BEEG. In Kapitel 3 werden die Ziele des Elterngelds jeweils getrennt für die drei Sphären identifiziert und ihre unterschiedlichen Zielhierarchien verglichen. Kapitel 4 analysiert die Valenz des Elterngelds in den Medien mit Regressionsanalysen. Dabei wird der Einfluss von Zieldefinition, Anlass, Zeitpunkt etc. daraufhin gemessen, ob Zeitungsbeiträge das Elterngeld positiv, ambivalent, neutral oder negativ bewerten.

1 Eine Zielhierarchie für den Bereich Wissenschaft lässt sich nicht empirisch robust feststellen (vgl. Kap. 3.3). 


\section{Elterngeld: Interaktion zwischen Politik, Medien und Wissenschaft}

\subsection{Die Entstehung des Elterngelds in der politischen Arena}

Der Willensbildungsprozess zur Entstehung des BEEG ist aus politikwissenschaftlicher Perspektive spannend: Die bereits in der rot-grünen Koalition vorbereitete Reform war erst in der Großen Koalition durchsetzbar, was sich durch eine spezielle Kombination von parteipolitischen Ursachen, Strategien politischer Akteure, wissenschaftlichen Kommissionen, verschobenen Verbandsinteressen und medialem Diskurs erklären lässt. Hier wird nicht der Willensbildungsprozess im Detail nachgezeichnet (hierfür siehe Henninger/ Wahl 2010; Mayer/Rösler in diesem Band), sondern vielmehr die Kommunikation der mit dem Elterngeld verbundenen Ziele analysiert. Dabei wird von der These ausgegangen, dass für alle diese Faktoren des Willensbildungsprozesses - also für Parteien, Akteure, Verbände, Wissenschaft und Medien - die Zielkonstellation von zentraler Bedeutung ist. Die komplementäre Zielstruktur des Elterngelds hat neuartige Interessenkoalitionen bewirkt: Zum einen entstand eine Interessenkoalition zwischen Feministinnen und Arbeitgeberverbänden, da das Elterngeld sowohl für Gleichstellung als auch für einen Anstieg der Müttererwerbstätigkeit als geeignetes Mittel gesehen wurde; zum anderen zwischen konservativen Strömungen, die sich mehr Geburten wünschen, und progressiven Kräften, die das Elterngeld aus sozialpolitischen Motiven unterstützen. Die ökonomischen und demografischen Argumente wurden von politischen Akteuren in der Öffentlichkeit platziert (vgl. Ristau 2005; Rürup/Schmidt 2003).

Die Bundesfamilienministerin Renate Schmidt hat auf der Klausurtagung des Bundeskabinetts in Bonn am 5.9.2004 den Vorschlag eines einkommensabhängigen Elterngelds in aller Deutlichkeit in die mediale Öffentlichkeit und gleichzeitig ins Zentrum der politischen Arena gebracht (Bannas 2004; Kamann 2004). ${ }^{2}$ Inhaltsanalysen mehrerer überregionaler Zeitungen wie Die Welt, Frankfurter Allgemeine Zeitung und Süddeutsche Zeitung belegen, dass diese Kabinettsklausur als Startschuss für die öffentliche Debatte fungiert, denn der Begriff „Elterngeld“ ist erstmals zu diesem Zeitpunkt im Titel bzw. überhaupt in den Archiven dieser Medien erwähnt. Knapp zwei Jahre später, am 20.6.2006, wurde der Gesetzentwurf der Fraktionen SPD sowie CDU/CSU eingebracht und am 25.8.2006 der Gesetzentwurf der Bundesregierung (Deutscher Bundestag 2006b, 2006c). Am 29.9.2006 wurde das BEEG im Bundestag verabschiedet und am 3.11.2006 im Bundesrat.

In den zwei Jahren und 24 Tagen, die zwischen Schmidts Vorschlag und dem Bundestagsbeschluss liegen, ist der Willensbildungsprozess v.a. von zwei Konfliktfeldern geprägt: Vätermonate und Verteilung. Ursprünglich war bei den Vätermonaten die 12minus-2-Variante geplant, wonach die vollen 12 Monate Elterngeld nur diejenigen Paare bekommen, bei denen der Vater mindestens zwei Monate Elternzeit nimmt. Dies wurde besonders in den Unionsparteien als Bevormundung von Paaren kritisiert, während in der SPD spezielle Anreize für Väter betont wurden. Gelöst wurde dieser Konflikt durch die

2 Seit Erfahrungen des Elterngelds in anderen Ländern wie Schweden vorliegen und speziell im Vorfeld dieser Kabinettsklausur war das Elterngeld zwar in einigen politischen Zirkeln ein Thema, zudem wurde es im wissenschaftlichen Diskurs thematisiert (siehe unten). Der zentrale Punkt ist hier jedoch, dass das Thema Elterngeld breit in die öffentliche Debatte und den öffentlich sichtbaren politischen Willensbildungsprozess eingeführt wurde. 
12-plus-2-Variante, die im Endeffekt eine Aufstockung des Zeitraums und der Kosten beinhaltet. Inhaltsanalysen von Medienberichten zufolge impliziert diese Variante, auf die sich der Koalitionsausschuss im Kanzleramt am 1.5.2006 einigte, statt einer Bestrafung (,Kürzung um zwei Monate“) einen Anreiz („,zwei Bonus-Monate“).

Die Verteilungsfrage war auch Gegenstand politischer Debatten, wobei die lohnabhängige Konzeption und deren Verteilungswirkung kritisiert wurden. Von der Fraktion Die Linke kritisierte beispielsweise Jörn Wunderlich eine „Besserstellung von Besserverdienenden“. Diese Kritik wurde jedoch nicht nur von Seiten der Opposition geäußert, sondern auch vom CDU-Ministerpräsidenten Jürgen Rüttgers. Rüttgers bezeichnete es als „,abwegig, denjenigen, die ein höheres Einkommen haben, mehr Geld zu geben“. ${ }^{3}$ Hier haben sich allerdings die Anhänger der lohnabhängigen Konzeption durchgesetzt, die Verteilungsfrage ist nach Verabschiedung des BEEG weniger heftig geführt worden als zuvor.

Die politische Diskussion um das Elterngeld nach 2007 ist v.a. im Kontext der fünf Ziele des Elterngelds zu interpretieren (dazu ausführlich Kap. 3).

\subsection{Der Siebte Familienbericht: Wissenschaft als Wegbereiter des Elterngelds?}

Die Wissenschaft hat gewissermaßen eine Doppelfunktion: Sie ist ein Feld mit eigenen Relevanzkriterien und fungiert andererseits als Akteurin. Bei der Entstehung des BEEG haben Akteure der Wissenschaft durch einzelne Gutachten (u.a. Bertram et al. 2005; Rürup/Schmidt 2003) eine begleitende Rolle und durch den Siebten Familienbericht (7. FB) (Deutscher Bundestag 2006a; vgl. Bertram et al. 2006) eine zentrale Rolle gespielt. Das BEEG ist neben dem Kinderbetreuungsausbau eine Komponente des Konzepts ,nachhaltige Familienpolitik“ (Rürup/Gruescu 2003; Bertram et al. 2005), das vom Bundesministerium für Familie, Senioren, Frauen und Jugend (BMFSFJ) mit enger Einbeziehung wissenschaftlicher Studien und Berater erarbeitet und kommuniziert wurde. Jun und Grabow (2008: 65) attestieren dem BMFSFJ Methoden, die partiell dem Evidence-Based-PolicyAnsatz entsprechen. Die Genese des 7. FB und der oben beschriebene politische Prozess fanden zeitlich parallel statt. Die Sachverständigenkommission des 7. FB wurde am 20.2. 2003 berufen, um bis Mitte 2005 einen Bericht vorzulegen; dieser wurde mit einer Stellungnahme der Bundesregierung am 26.4.2006 veröffentlicht. Inhaltlich betont der 7. FB zwei Elemente, die für das BEEG zentral sind: die Lebensverlaufsperspektive und die Zeitpolitik (ibd.: 260f.). Das Elterngeld wird an drei Stellen des 7. FB - mit zunehmender Intensität - aufgegriffen: Als positives Beispiel aus dem europäischen Vergleich (ibd.: 64), als Instrument einer neuen Balance einer nachhaltigen Familienpolitik, die den „Widerspruch zwischen beruflicher Selbstständigkeit und ökonomischer Abhängigkeit vom Ehemann" auflösen hilft (ibd.: 250f.), und ausführlich als eine der zentralen Zukunftsvisionen der Kommission (ibd.: 284-289).

„Im Grundsatz handelt es sich bei diesem Modell [Erziehungsgeld] immer noch um das klassische Modell der Versorgerehe, das davon ausgeht, dass Mutter und Kind während der Phase der frühesten Kinderbetreuung sich in die ökonomische Abhängigkeit vom Mann begeben oder je nach Bedürftigkeit durch den ,Vater Staat‘ unterstützt werden.“ (Deutscher Bundestag 2006a: 285)

3 Quelle beider Zitate: FAZ vom 16.1.2006, S. 13 und FAZ vom 2.12.2005, S. 2. 
„Die nordeuropäischen Modelle [Elterngeld] mit ihrer Konzeption eines eigenständigen Einkommens sind eine zukunftsorientierte Variante der ökonomischen Basis von Ehe und Familie. Darüber hinaus sind die nordeuropäischen Modelle so konstruiert, dass Familie und Kinder in die Entscheidungslogik der Lebensläufe junger Erwachsener passen.“ (ibd.: 287)

Diese beiden Zitate verdeutlichen die Kernargumentation des Abschnitts „Zukunftsszenarium: Finanzielle Transfers im Lebensverlauf", bei dem das einkommensabhängige Elterngeld empfohlen wird. Dabei werden dem deutschen Erziehungsgeld im Kontext weiterer sozialpolitischer Leistungen das schwedische und das finnische Modell des Elterngelds gegenübergestellt. Während das schwedische Modell einen 80\%-Lohnersatz und eine dreimonatige Übergangszeit vorsieht, wird für Deutschland eher das finnische empfohlen, das mit $67 \%$ Lohnersatz und wahlweise Krippenbesuch oder Betreuungsgeld bis zum 36. Monat tatsächlich als Vorbild der deutschen Familienpolitik seit 2006 gelten kann. Die zentralen Argumente pro Elterngeld sind neben ökonomischer Unabhängigkeit von Frauen und moderner Lebenslaufspolitik, dass es der impliziten „Abwertung der Fürsorgeleistungen" (ibd.: 288) entgegenwirkt. Dazu kommt die ökonomische Argumentation, wonach das Elterngeld die Schwankung des Einkommens reduziert und langfristig durch kontinuierliche Erwerbsbiografien die Alterssicherung stärkt.

„Erstmals hat eine Sachverständigenkommission schon während der Erarbeitung intensiv den Dialog mit relevanten gesellschaftlichen Akteuren gesucht" (Deutscher Bundestag 2006a: 29).

Die erhebliche Wirkung des 7. FB basiert auch auf dem Dialog der Kommission mit Arbeitgebern, Gewerkschaften, Kirchen und Familienverbänden. Vor allem aber ist die inhaltliche und zeitliche Parallelität zwischen Bericht und Familienpolitik entscheidend, die auf eine konstruktive Abstimmung der Akteure hindeutet. Inwieweit der 7. FB das Elterngeld mitinitiiert hat oder inwiefern er von den politischen Akteuren, die das Elterngeld wollten, im Willensbildungsprozess als Begründungshilfe genutzt wurde, kann hier nicht eingeschätzt werden. Das Elterngeld ist in der Wissenschaft länger bekannt, der 7. FB hat das Verdienst, es popularisiert zu haben. Bezüglich der Umsetzung des Elterngelds hat die Strategie des 7. FB, eine konkrete Politikmaßnahme in einer für Deutschland umsetzbaren Variante zu empfehlen, sich als hilfreich erwiesen. Der Einfluss des 7. FB basiert folglich aus einer Kombination aus Timing, Argumentation und Dissemination.

\subsection{Anlässe für eine Berichterstattung zum Elterngeld in den Medien}

In den Medien hat das Elterngeld eine große Aufmerksamkeit erfahren. Medien berichten über bestimmte Ereignisse bzw. Anlässe wie die Publikation neuer Studien, politische Debatten oder juristische Entscheidungen und sie kommentieren diese. Dabei kommt ihnen eine Doppelrolle zu: Einerseits beeinflussen politische Entscheidungen, wissenschaftliche Befunde oder Rechtsprechung via Medien die öffentliche Meinung zum Elterngeld (Medien fungieren hier als Transporteure), andererseits beeinflussen die Medien maßgeblich die öffentliche Meinung - nicht nur durch Kommentare, sondern auch durch Auswahl und Nuancierung der Berichterstattung (Medien als Akteure).

In diesem Abschnitt werden die unterschiedlichen Anlässe für eine Berichterstattung zum Elterngeld in den Medien analysiert. Dies basiert auf einer Inhaltsanalyse, die später auch verwendet wird, um die Auswahl der Ziele (Kap. 3.2) und die Bewertung des El- 
terngelds im Zeitverlauf (Kap. 4) zu erforschen. Bei der Inhaltsanalyse werden beide Methoden Valenzanalyse und Frequenzanalyse kombiniert (vgl. Krippendorff 2004; Schnell et al. 2005). Grundlage ist die Berichterstattung der Frankfurter Allgemeinen Zeitung (FAZ) inklusive Frankfurter Allgemeine Sonntagszeitung (FAS) und Internetberichterstattung (FAZ.NET) von 2004 bis 2012. Die FAZ ist eine der am meisten verbreiteten und renommiertesten Tageszeitungen in Deutschland und lässt sich dem konservativen Spektrum zuordnen. Die Auswahl nur einer Zeitung folgt pragmatischen Gründen und dabei der Annahme, dass sie keine journalistische Einseitigkeit im Kontext des Elterngelds aufweist. Voranalysen u.a. mit der Süddeutschen Zeitung haben gezeigt, dass sich sowohl die Anlässe der Berichterstattung als auch die Zielzuschreibungen ähneln. ${ }^{4}$ Demzufolge ist es plausibel, dass die Berichterstattung in der FAZ zum Elterngeld vom Prinzip her charakteristisch für die deutsche Zeitungslandschaft ist. Eine Ausweitung auf mehrere Zeitungen würde zwar die Fallzahl erhöhen, nicht jedoch die Relationen von Anlässen und Zielattribuationen grundsätzlich verändern. Einbezogen wurden sämtliche Beiträge in FAZ, FAS und FAZ.NET, bei denen das Wort „Elterngeld“ im Titel oder Untertitel enthalten ist - insgesamt 176 Artikel von frühestens am 5.9.2004 bis Ende 2012. Zeitlich gesehen handelt es sich um eine Vollerhebung aller für das Elterngeld potenziell besonders relevanten Artikel (Überschrift-Kriterium).

Die Anlässe der jeweiligen Artikel wurden in sechs Kategorien unterteilt, die dem oben skizzierten Kontext von Politik, Wissenschaft und Medien entsprechen. Die politische Arena ist hier nach genuin politischen Akteuren und Verbänden aufgeteilt, der Bereich Wissenschaft zwischen amtlichen Statistiken des Statistischen Bundesamtes und anderen Studien. Ferner gibt es zwei Medien-Kategorien mit dem Kommentar, bei dem die Zeitung im direkten Sinne als Meinungsmacher fungiert, und mit Artikeln zur Information über die Ausgestaltung sowie gesetzliche Auslegungen des Elterngelds. Die Zuweisung der Kategorien erfolgte gegenseitig ausschließend. ${ }^{6}$ Abbildung 2 zeigt, dass der Anlass für fast die Hälfte der Artikel (49,4\%) der politische Diskurs ist. Besonders viele derartige Artikel gibt es in der Phase der parlamentarischen Willensbildung, die mit der Konkretisierung der Elterngeldpläne durch die damalige Bundesfamilienministerin Renate Schmidt (August 2005) beginnt und bis zum Bundestagsbeschluss zur Einführung der Elterngelds am 29.9.2006 geht. Nach Einführung des BEEG zeigt die Medienanalyse auch Phasen der politischen Diskussion, die beispielsweise durch Kürzungsvorschläge wie in den Haushaltsverhandlungen im Sommer 2010 veranlasst sind oder die von CDU-CSU-Fraktionschef Volker Kauder (2012) durch ein Interview mit der Süddeutschen Zeitung initiierte Diskussion über die Wirksamkeit des Elterngelds.

4 Hier sei betont, dass insbesondere die häufige Orientierung am Ziel „Geburtenrate steigern“ kein Phänomen nur konservativer Zeitungen ist. Für zukünftige Analysen wäre allerdings ein Vergleich der Valenz von Kommentar-Beiträgen verschiedener Zeitungen sinnvoll.

5 Der Titel des ältesten Artikel: „Schröder: Der Umbau des Sozialstaates wird fortgesetzt“, Untertitel: „Klausurtagung in Bonn / Elterngeld statt Erziehungsgeld“.

6 In den Fällen, bei denen beispielsweise Zahlen des StBA und politische Reaktionen darauf Gegenstand des Artikels waren, wurde der (zeitlich) primäre Anlass zugewiesen. 
Abbildung 2: Zeitungsartikel zum Elterngeld 2004-2012 und ihr Anlass

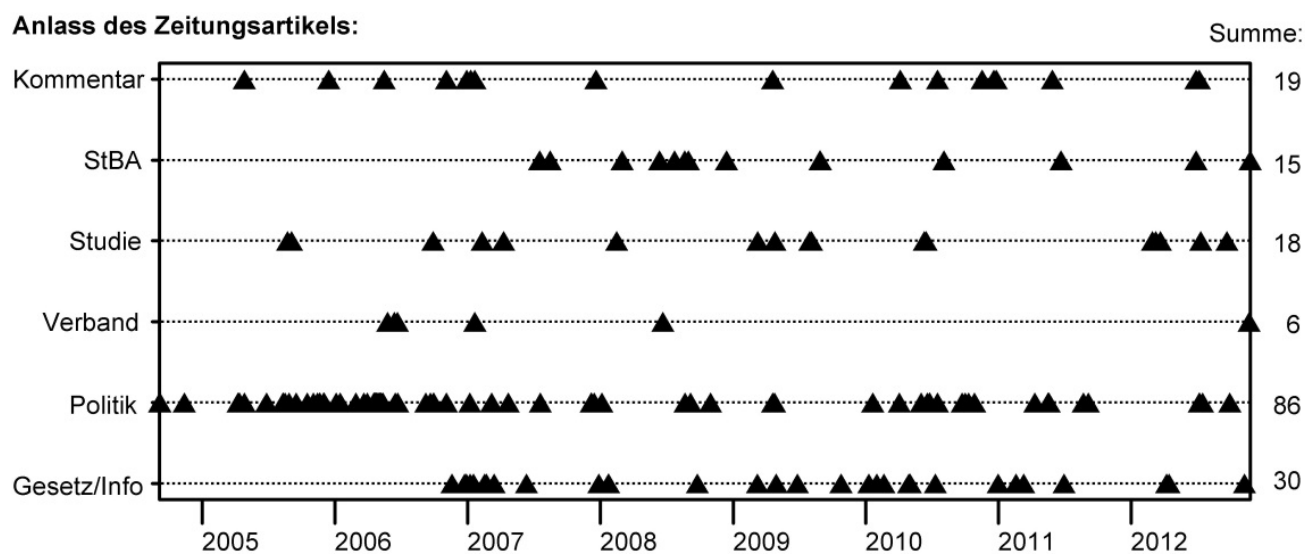

Anmerkungen: Die Summe der analysierten Artikel beträgt 176, wobei hier nur 174 berücksichtigt sind, da die anderen beiden einen sonstigen Anlass haben. StBA = Statistisches Bundesamt.

Quelle: Eigene Berechnung und Darstellung.

Bemerkenswert für den politisch-medialen Prozess in Deutschland ist, dass das Elterngeld nur selten durch Verbände auf die Agenda gesetzt wurde. Die wenigen derartigen Artikel sind auf Presseerklärungen der Bundesvereinigung der Deutschen Arbeitgeberverbände (BDA) oder Gremien der Katholischen Kirche zurückzuführen - auch wenn es eine Vielzahl an Familienverbänden gibt, die das Elterngeld mit Presseerklärungen begleiten. Diese geringe Medienpräsenz belegt die schwach ausgeprägte familienpolitische Interessenvertretung durch Verbände, der strukturelle Ursachen wie eine geringe Organisationsfähigkeit und Schwierigkeiten der Mobilisierung von Familien zugrunde liegen (zu verhandlungstheoretischen Schwächen vgl. Gerlach 2004: 359). Dazu können mediale Selektionskriterien eine Rolle spielen. Bemerkenswert ist die Tatsache, dass der auffälligste Verband in der medialen Diskussion um das Elterngeld die BDA ist, die nicht die Interessen von Familien, sondern die von Arbeitgebern vertritt.

Wissenschaftliche Anlässe machen 19\% der Elterngeldartikel aus, 8,6\% beruhen auf Mitteilungen des Statistischen Bundesamtes und 10,4\% auf wissenschaftlichen Studien. Beide Anlässe sind kontinuierlich über die Zeit verteilt, wobei es eine leichte Häufung im Sommer 2008 gibt, nachdem zum ersten Mal Zahlen der Elterngeldstatistik für den kompletten Zeitraum von 14 Monaten für die Anfang 2007 geborenen Kinder vorliegen. Die meisten wissenschaftlichen Anlässe sind mit den Themen Väterbeteiligung und Geburtenentwicklung verbunden. 


\section{Unterschiedliche Zielhierarchien des Elterngelds in Politik, Wissenschaft und Medien}

\subsection{Ziele in der Politik}

Um die Ziele der Politik zu analysieren, kann man sich auf zwei unterschiedliche Arten von Quellen berufen: Bundestagsdrucksachen und Politiker-Zitate. Im Gesetzentwurf der Bundesregierung (bzw. dem der Fraktionen SPD und CDU/CSU) werden Ziele explizit bzw. implizit genannt, die sich in fünf Kategorien einteilen lassen. Die Analyse der Bundestagsdrucksachen wie Gesetzentwurf, Fraktions- und Ausschussberichte vor Verabschiedung des BEEG (Deutscher Bundestag 2006b, 2006c, 2006d, 2006e, 2006f) ermöglicht es anhand der Häufigkeit der genannten Ziele, eine Hierarchie der Ziele zu erkennen, wobei Einkommensaspekte am Anfang und demografische Aspekte am Schluss stehen.

1. Einkommen in der Familiengründungsphase sichern

„In Deutschland steht Familien dann am wenigsten Geld zur Verfügung, wenn die Kinder am kleinsten sind. Familiengründungen und das Aufziehen von Kindern bewirken Einkommenseinschränkungen, die vor allem durch Erwerbsunterbrechungen verursacht werden. [...] Das Elterngeld will dazu beitragen, dass es beiden Elternteilen auf Dauer besser gelingt, ihre wirtschaftliche Existenz zu sichern.“ (Deutscher Bundestag 2006c: 1f.)

2. Zeit und Schonraum im 1. Jahr ermöglichen

„Das Elterngeld unterstützt Eltern in der Frühphase der Elternschaft und trägt dazu bei, dass sie in diesem Zeitraum selbst für ihr Kind sorgen können. Es eröffnet einen Schonraum.“ (Deutscher Bundestag 2006c: 1)

3. Steigende Erwerbsbeteiligung von Müttern fördern

„Das bisherige Bundeserziehungsgeld (...) begünstige längere Erwerbsunterbrechungen der Frauen und werde damit der wachsenden Berufsorientierung der Frauen nicht mehr gerecht." (Deutscher Bundestag 2006d: 2)

4. Gleichstellung: Steigende Fürsorgebeteiligung von Vätern fördern

„Beruf und ein Leben mit Kindern sind Bestandteile der Lebensplanung der Mehrzahl junger Frauen und Männer. Tatsächlich kehren aber die meisten Frauen erst in den Beruf zurück, wenn die Kinder älter sind, und nur fünf Prozent der Väter gehen in Elternzeit.“ (Deutscher Bundestag 2006c: 1)

5. Fertilität-Familiengründung erleichtern

„Die Bundesregierung richtet ihre familienpolitischen Leistungen neu aus, um (...) den Menschen mehr Mut zu mehr Kindern zu machen.“ (Deutscher Bundestag 2006c: 2)

Die Ziele Gleichstellung und Wahlfreiheit lassen sich den Kategorien 3 und 4 zuordnen und mit Beteiligungsquoten in Fürsorge- und Erwerbsarbeit (in Vergleich zu den Präferenzen) empirisch überprüfen. Die Erwerbsbeteiligung der Frauen zu steigern ist zudem ein Ziel, das nicht nur aus Gleichstellungsmotiven, sondern auch aus Sicht der Arbeitgeber verfolgt wird. Das Ziel der Erhöhung der Geburtenrate ist im Gesetzestext nur indirekt formuliert wie „Mut zum Kind“ oder „Familiengründung erleichtern“. Deutlicher ist der Hinweis auf diese Zieldimension in der Problembeschreibung des BEEG, bei dem die niedrige Geburtenrate Deutschlands und die hohe Kinderlosigkeit thematisiert werden. 
Im Entschließungsantrag der FDP werden die Mehrdimensionalität der Zielsetzung und auch ihr Fünfklang besonders deutlich:

„Der vorliegende Gesetzentwurf verbindet hingegen verschiedene Zielsetzungen der Familien-, Gleichstellungs-, Bevölkerungs-, Arbeitsmarkt- und Sozialpolitik.“ (Deutscher Bundestag 2006f: 1)

Im Bericht des Haushaltsausschusses wird die Einführung des Elterngelds mit dem ersten Ziel begründet, „um Familien bei der Sicherung ihrer Lebensgrundlage zu unterstützen, wenn sich die Eltern vorrangig um die Betreuung ihrer Kinder kümmern." (Deutscher Bundestag 2006e: 1) Darüber hinaus wird im Absatz zu den Auswirkungen auf die Wirtschaft das dritte Ziel betont: „Eine steigende Erwerbsbeteiligung von Frauen hilft, ein ansonsten aufgrund der demographischen Veränderungen sinkendes Angebot insbesondere von Fachkräften auf dem Arbeitsmarkt auszugleichen." (ibd.: 2)

In späteren Bewertungen (und Verteidigungen) des Elterngelds stützt sich das BMFSFJ manchmal auch auf den Fünfklang der Ziele. So schätzt Bundesfamilienministerin Ursula von der Leyen mit Verweis auf alle fünf Ziele ein, dass das BEEG „ein uneingeschränkter Erfolg“" sei (von der Leyen 2008).

Während das Ziel Geburtensteigerung in den Gesetzestexten nur indirekt bzw. als den sozialpolitischen Zielen (Ziel 1 und 2) nachgeordnet kommuniziert wird, finden sich in Äußerungen von einzelnen Politikern klare Formulierungen zu geburtensteigernden Zielen des Elterngelds:

„(Kanzler Schröder) verwies darauf, dass v.a. Akademikerinnen immer seltener Kinder bekommen
wollten. Eine solche Entwicklung habe gesellschaftliche Folgen, warnte er.“
Bundeskanzler Gerhard Schröder (2004, indirektes Zitat)

„Das zweite Motiv für die Einführung des Elterngeldes war ohne Zweifel die Steigerung der Geburtenrate in Deutschland."

Bundesfamilienministerin i.R. Renate Schmidt (2012)

Diese Zitate verdeutlichen, dass das Ziel höherer Geburtenraten auf höchster politischer Ebene - Bundeskanzler und zuständige Ministerin - mit dem Elterngeld verbunden war. Beide maßgeblichen Akteure verwiesen jedoch auch auf die anderen Ziele. Eine Priorisierung des demografischen Ziels gegenüber anderen Zielen, wie sie Georg Milbradt vorgenommen hat, ist eher die Ausnahme. Jedoch gibt es viele Politiker, die diesem Ziel keine explizite Rolle zusprechen. Daher lässt sich zweierlei konstatieren: (1) Das geburtensteigernde Ziel des Elterngelds ist in der Politik kein Tabu, es wird jedoch nur von einem Teil der Akteure vertreten und in Gesetzestexten nur indirekt angesprochen. (2) Mit dem Elterngeld hat der Gesetzgeber fünf Ziele verbunden, denen unterschiedliche Wichtigkeit zugesprochen wird, wobei Einkommensaspekte am Anfang und Fertilitätsziele am Ende der Prioritätsrangfolge stehen.

7 Zitiert nach: FAZ vom 6.9.2004, S. 4. Das Zitat ist anlässlich der Klausurtagung des Kabinetts in Bonn am 5.9.2004 gefallen, bei dem die rot-grüne Bundesregierung erstmals beschloss, das Elterngeld anzugehen.

8 Zitiert nach: Wolber (2006). 


\subsection{Ziele in den Medien}

Die Bewertung des Elterngelds hängt auch davon ab, welche Ziele mit dem BEEG verbunden und als zentral erachtet werden. Die These ist, dass eine grundsätzliche Verzerrung der Zielattribuation zwischen Politik und Medien den Diskurs des deutschen Elterngelds kennzeichnet. Um dies zu überprüfen, wird eine Frequenzanalyse von Zielnennungen in den Medien vorgenommen. Dabei werden auch implizite Ziele in den Artikeln identifiziert, was eine manuelle Codierung erforderlich macht. Die Erfassung expliziter Ziele mit reinen computergestützten Frequenzanalysen von auftretenden Begriffen wäre ungeeignet. Das hier gewählte Kategorienschema folgt theoretischen Erwägungen und speziell der Auswahl der fünf in der Analyse der Bundestagsdrucksachen identifizierten Ziele: Geburtenrate erhöhen, Gleichstellung, Müttererwerbstätigkeit steigern, Schonraum und Einkommen sichern. Ein Pretest hat bestätigt, dass sich sämtliche Zielnennungen auf diese fünf Zielkategorien verdichten lassen und die Reliabilität durch genaue und stabile Zuordnungen gewährleistet ist (vgl. Krippendorff 2004: 211ff.). Das methodische Kriterium, wonach die Kategorien umfassend sind und einander ausschließen (vgl. Schnell et al. 2005: 411), ist erfüllt. Pro Artikel wird jedes Ziel nur einmal gezählt, es können aber mehrere Ziele vorkommen. Zudem hat exakt die Hälfte der Artikel keine Zielattribuationen, was häufig auf solche mit neutraler Valenz gegenüber dem Elterngeld zutrifft. Bei nur drei Artikeln wurden alle fünf Ziele genannt; drei Mal sind es vier Ziele, 12 mal drei, 27 mal zwei und in 42 Artikeln wird nur ein Ziel genannt.

Am häufigsten wird das Ziel einer Steigerung der Geburtenrate genannt (28,3\% aller Zielnennungen), es folgen die Sicherung des Einkommens (24,5\%), Gleichstellung $(23,3 \%)$, Müttererwerbstätigkeit (12,6\%) sowie Zeit bzw. Schonraum im ersten Lebensjahr des Kindes (11,3\%). Bemerkenswert ist, dass alle fünf Ziele zu einem zweistelligen Prozentsatz genannt werden, also im medialen Diskurs wahrgenommen werden. Da keine weiteren Ziele in den Inhaltsanalysen auftauchen, lässt sich der Fünfklang der Zielstruktur des Elterngelds bestätigen. Das dominierende Ziel ist dabei zweifellos die Geburtenrate (siehe Abbildung 3). 
Abbildung 3: Frequenzanalyse: Zielnennungen in Zeitungsartikeln zum Elterngeld 2004-2012

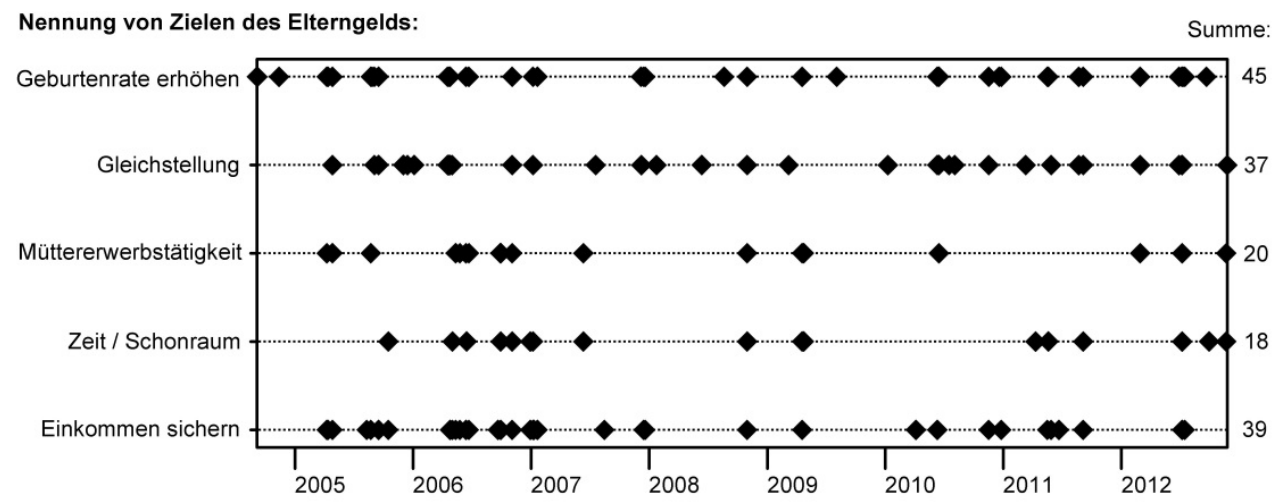

Quelle: Eigene Berechnung und Darstellung.

Die Frequenzanalyse von Zielnennungen ermöglicht auch Aufschlüsse über Veränderungen im Zeitverlauf. Betrachtet man die Zielnennungen in den drei Dreijahresphasen 200406, 2007-09 und 2010-12, fällt auf, dass Einkommen und Müttererwerbsquote im Zeitverlauf weniger genannt wurden und Geburtenrate und Gleichstellung zunehmend wichtiger wurden. Vor Einführung des Elterngelds wurde das Einkommen mit 26,8\% sogar häufiger genannt als die Geburtenrate mit 25,4\%. Im letzten Zeitraum 2010-2012 haben Fertilitätsziele $(33,3 \%)$ und Gleichstellung $(27,8 \%)$ die Zielattribuierung des Elterngelds dominiert.

\subsection{Ziele in der Wissenschaft}

Die Zieldefinitionen der wissenschaftlichen Gutachten und Essays, die bei der Entstehung des BEEG einflussreich waren, sind heterogen. Dies zeigt auch der Vergleich zwischen dem 7. FB und dem einflussreichen Rürup-Gutachten.

„Das [Elterngeld als Lohnersatz] bedeutet auch, dass die Entscheidung für ein Kind für eine Frau nicht mit der gesellschaftlichen Erwartung verknüpft ist, die eigene ökonomische Selbstständigkeit aufgeben zu müssen.“ (7. FB: 64)

Der 7. FB hat als Ziele in besonderer Weise Einkommenssicherung und Gleichstellung betont. Einkommenssicherung bezieht sich dabei auf die Reduzierung von Kinderarmut (ibd.: 286) und Glättungen im Lebensverlauf, um den „Achterbahneffekt“ zu reduzieren. Das Ziel Gleichstellung wird hier durch die ökonomische Unabhängigkeit der Frau verfolgt und weniger als im späteren Diskurs durch das Mittel der Partnermonate. Mit dem Verweis auf das Humankapital, das im Fünften Familienbericht in den Mittelpunkt gestellt wurde, lassen sich indirekt auch die Ziele Geburten und Müttererwerbstätigkeit verbinden. Die letztgenannten beiden Ziele sind explizit und prioritär von Gruescu und Rürup mit dem Konzept der nachhaltigen Familienpolitik verbunden, bei dem das Elterngeld als ein zentrales Instrument fungiert. 
„Nachhaltige Familienpolitik strebt die Erhöhung der Geburtenrate und eine Steigerung der Erwerbstätigkeit von Frauen an.“ (Gruescu/Rürup 2005: 3)

Für eine Sekundäranalyse von Zielen in wissenschaftlichen Studien zu den Wirkungen des Elterngelds sind die vorhandenen Analysen - v.a. mit Peer-Review - zu gering. Maßgeblich für die Auswahl von analysierten Zielen, also die Festlegung der abhängigen Variablen, sind Datenverfügbarkeit, Operationalisierungsmöglichkeiten, theoretische Erwägungen, die Erwartung von Veränderungen bestimmter Variablen und Drittmittelaufträge. Letzteres wird evident, da ein erheblicher Teil bisheriger Forschung durch die von der Bundesregierung initiierte Evaluation finanziert wurde. Hier wurde das Elterngeld hinsichtlich seiner Wirkung auf drei Ziele analysiert: (1) Schonraum, der als Abwesenheit „finanzieller Nöte“ definiert ist, (2) die mittel- und langfristige Sicherung der wirtschaftlichen Existenz beider Eltern, v.a. durch einen schnelleren beruflichen Wiedereinstieg von Müttern und (3) eine Erhöhung der „Väterbeteiligung an der Kinderbetreuung“ (vgl. Wrohlich et al. 2012). Kluve und Tamm (2009) nennen hier vier Ziele. Grundsätzlich sind wissenschaftliche Analysen nicht an umfassende Zieldefinitionen gebunden, gelegentlich liegt der Fokus auf nur einer abhängigen Variablen. Allerdings werden wissenschaftliche Befunde vielfach über die Medien in die breite Öffentlichkeit getragen (vgl. Abschnitt 2.3).

\section{Valenz des Elterngelds in den Medien}

Der Fokus auf unterschiedliche Ziele im Zeitverlauf, die man grafisch gut aus Abbildung 3 ablesen kann, hat einen starken Einfluss auf die Valenz der Berichterstattung. Dies mag an einigen Beispielen erläutert werden: Die Veröffentlichung neuer Daten zur Geburtenentwicklung wurde gelegentlich zum Anlass für eine mediale Bewertung des Elterngelds, selbst wenn es sich um absolute Geburtenzahlen gehandelt hat, die primär mit der Altersstruktur der Frauen in der Bevölkerung zusammenhängen. Der zu erwartende kontinuierliche Rückgang der absoluten Geburtenzahlen kann folglich jedes Jahr zu einer negativen Valenz des Elterngelds führen - auch wenn der Zusammenhang inhaltlich unplausibel ist. Ein weiteres Beispiel betrifft Zeitungsartikel, die Daten zu Vätermonaten zum Anlass nehmen. Die steigende Beteiligung von Vätern an der Elternzeit, die sich deutlich vom niedrigen Niveau von 3,5\% vor dem BEEG abhebt, wird im Kontext des Ziels Gleichstellung mit einer positiven Valenz verbunden.

Die Valenz wird operationalisiert, indem die implizite oder explizite Bewertung eines jeden Artikels gegenüber dem Elterngeld als positiv, negativ oder ambivalent (= positiv und negativ gleichzeitig) eingestuft wurde. ${ }^{9}$ Die Korrelation zwischen Valenz und der Summe der genannten Ziele ist hochsignifikant ${ }^{10}$ mit einem Pearson's $r$ von 0,408. Dieser Zusammenhang lässt sich auf folgende These fokussieren: Je mehr Ziele berücksichtigt werden, desto positiver ist die Berichterstattung. Dieser Zusammenhang ist auch in multivariaten Modellen stabil, also unabhängig von Zeitpunkt oder Anlass des Artikels. Von

9 Zum Umgang mit neutralen Valenzen: siehe unten.

10 Das Adjektiv „hochsignifikant“ entspricht einer Irrtumswahrscheinlichkeit von $<0,01$, „signifikant“ einer von $<0,05$ (zweiseitiger Test). 
den einzelnen fünf Zielen sind nur Zeit/Schonraum und Müttererwerbstätigkeit hochsignifikant mit einer positiven Valenz assoziiert $(r=0,324$ bzw. 0,332). Das Ziel Gleichstellung wird in den Medien tendenziell positiv bewertet, jedoch ist dieser Zusammenhang insignifikant. Dies könnte auf die kontroverse parteipolitische Willensbildung bezüglich expliziter Vätermonate (12-2 bzw. 12+2) in den Jahren 2005-2006 zurückzuführen sein. Ein Zusammenhang von demografischen Zielen und negativer Valenz lässt sich nicht feststellen.

Die Valenzanalyse von Zeitungsartikeln zum Elterngeld zeigt, in welchem Ausmaß das Elterngeld bewertet wird und in welcher Richtung diese Bewertungen ausfallen (siehe Abbildung 4). Während 52,8\% der Berichterstattung keine explizite oder implizite Bewertung aufweisen, ist knapp die Hälfte mit einer spezifischen Valenz verbunden. Diese Bewertungen sind relativ gleichmäßig verteilt, wobei es etwas mehr Artikel mit positiver Valenz gibt $(34,9 \%)$ als solche mit negativer $(25,3 \%)$ und viele Artikel beide Valenzen aufweisen, sodass sie als ambivalent klassifiziert werden $(39,8 \%)$.

Abbildung 4: Valenzanalyse von Zeitungsartikeln zum Elterngeld 2004-2012

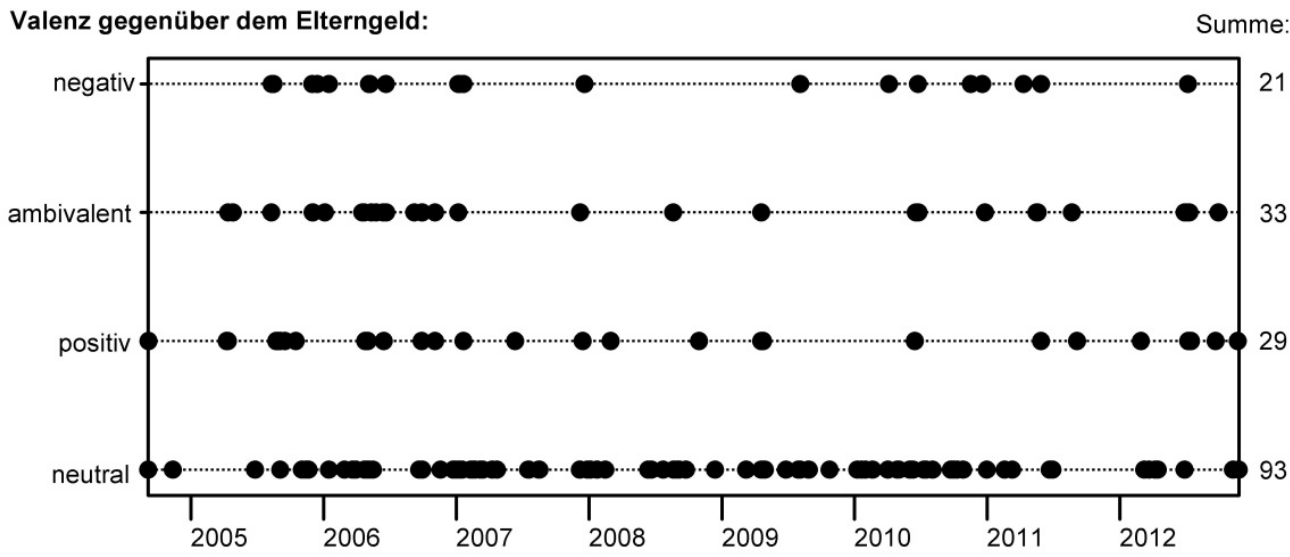

Quelle: Eigene Berechnung und Darstellung.

Hat sich die Valenz der Berichterstattung zum Elterngeld im Zeitverlauf geändert? Um dieser Frage nachzugehen, wurden drei Zeiträume separat betrachtet: Der Zeitraum vor Einführung des BEEG (Z_2004-06), der unmittelbar danach (Z_2007-09) und die letzten drei Jahre (Z_2010-12). In Z_2004-06 waren mehr Artikel mit Valenz und nur 36,5\% neutral, während danach die Neutralen in der Mehrheit waren. Vor Einführung des Elterngelds war die Valenz überdurchschnittlich oft ambivalent $(47,6 \%$ der bewertenden Artikel). Beides lässt sich dahingehend interpretieren, dass die Umstrittenheit vor der Einführung größer war als danach. Dies kann jedoch schlicht mit dem politischen Prozess begründet werden, da in Z_2004-06 die parlamentarische Gesetzgebungsphase stattfand und ein Paradigmenwechsel wie das Elterngeld ein kontroverses Medienecho hervorruft. In den drei Jahren nach der Einführung waren 71,4\% der Berichte neutral, in Z_2010-12 nur 52,6\%, was darauf hinweist, dass das Elterngeld inzwischen wieder etwas stärker umstritten ist. Dabei ist jedoch die medienimmanente Logik zu berücksichtigen, wonach 
Medien besonders an Kontroversen interessiert sind. ${ }^{11}$ Bemerkenswert ist, dass in allen drei Phasen die positive Valenz die negative überwogen hat, wobei der Vorsprung der positiven Valenz im Zeitverlauf etwas nachgelassen hat. ${ }^{12}$

Auch der Zusammenhang zwischen den unterschiedlichen Anlässen der Berichterstattung und der Valenz wurde bi- und multivariat untersucht. Nur bei zwei Anlässen zeigt sich bivariat ein signifikanter Zusammenhang: Wissenschaftliche Studien sind mit einer positiven Valenz der medialen Berichterstattung assoziiert $(r=0,224)$, genuine Kommentare zeitigen systematisch eine negative Bewertung $(r=0,259)$. Bei den Anlässen Politik, Presseerklärung des Statistischen Bundesamtes und Gesetzinformation ist dem Vorzeichen nach ein insignifikanter positiver Zusammenhang mit einer positiven Valenz gegeben.

In der multivariaten Analyse werden Determinanten der Valenz mit der linearen Regressionsmethode (OLS) untersucht. Bezüglich der abhängigen Variablen „Valenz “"13 wurde das Sample von 176 Fällen auf 83 Fälle reduziert, um Artikel mit neutraler Valenz nicht mit ambivalenten zu mischen. Es werden also nur die Artikel der Medienanalyse berücksichtigt, die deutliche Bewertungen aufweisen. Hintergrund aus statistischer Sicht ist, dass die hohe Zahl neutraler Artikel die Analyse der Determinanten der Valenz verzerren würde. Inhaltlich ist diese erhebliche Reduzierung des Samples plausibel, da es darum geht, die Tendenz von Bewertungen (und nicht ihr Ausbleiben) gerade im Kontext von Zielen und Anlässen zu verstehen. Neutrale Artikel sind keineswegs die Mitte zwischen positiver und negativer Valenz (was dagegen für ambivalent gilt), sondern Teil einer dualen Kategorie „neutral“ versus ,bewertend“. Wer dieser Argumentation nicht folgen möchte, findet im Anhang zum Vergleich eine Regressionsanalyse für das Gesamtsample mit der Kodierung von Artikeln mit neutraler Valenz entsprechend der mit ambivalenter Valenz; dieser Vergleich zeigt, dass das Vorgehen an den zentralen Befunden wenig ändert. Als unabhängige Variable werden das Jahr des Artikels, der Anlass des Artikels, die Nennung einzelner Ziele als Dummies und die Summe der genannten Ziele verwendet.

Auf den Umgang mit potenziellen methodischen Problemen der Regressionsanalyse sei hier kurz hingewiesen: Multikollinearität ist nur bei der Variablen „Anlass Politik“ in Modell 1 gegeben, ansonsten nicht, wie die Interkorrelationsmatrix zeigt. Hier wird der Konvention gefolgt, Interkorrelationen bis $0,7 \mathrm{zu}$ tolerieren und bei solchen über dieser Schwelle Variablen auszuschließen (vgl. u.a. Wagschal 1999: 237). Das Problem der Heteroskedastizität der Residuen ist nicht gegeben, da die Varianz der Residuen konstant ist. Das dritte potenzielle Problem bei Regressionsanalysen mit derartigen Fallzahlen betrifft die Ausreißer; aufgrund der begrenzten Spannbreite der abhängigen Variablen kann ausgeschlossen werden, dass Ausreißer die Befunde verzerren.

Tabelle 1 zeigt die Befunde der Regressionsanalyse. Modell 1 ist das Best-fit-Modell mit einem korrigierten Determinationskoeffizienten von 0,275. Die Valenz ist demnach umso positiver, je mehr Ziele im Artikel genannt sind, wenn der Anlass kein Kommentar ist, wenn der Anlass eine wissenschaftliche Studie ist, wenn das Ziel des Schonraums für

11 Dazu kommt, dass hier nur Artikel mit dem Begriff Elterngeld in den Überschriften verwendet wurden, auch um auf Kontroversen und Bewertungen zu fokussieren.

12 In Z_2004-06 waren Artikel mit positiver Valenz 1,62-fach häufiger als solche mit negativer, in Z 2007-09 war es 1,40-fach und in Z 2010-12 1,13-fach.

13 Kodierung der AV: 1 = negativ; 2 = ambivalent; 3 = positiv. 
Eltern genannt wird und kein Fertilitätsziel genannt wird. Ein schlankes Erklärungsmodell bietet Modell 4, das die drei erklärungskräftigsten Determinanten berücksichtigt.

Für Tabelle 1 wurden neben dem Best-fit- und dem schlanken Modell noch zwei weitere ausgewählt, welche die Variation des Einflusses in Abhängigkeit von verschiedenen Modellspezifikationen verdeutlichen: Während die Variable „Summe der Ziele“ in allen Modellen hochsignifikant ist und T-Werte zwischen 2,7 und 4,7 aufweist, sind die Variablen „Anlass Medien-Kommentar“ und „Anlass Studie“ nur in einzelnen Modellen (hoch)signifikant. Davon abgesehen ist der Zusammenhang zwischen Kommentar und negativer Valenz über viele, auch hier nicht gezeigte, Modelle, robust. Der Zusammenhang zwischen Studie und positiver Valenz schwankt um das einfache Signifikanzniveau (Irrtumswahrscheinlichkeit $<5 \%$ ), der T-Wert schwankt zwischen 1,5 und 2,1. Bemerkenswert ist, dass die Vorzeichen der Anlass-Variablen stabil sind und sämtliche Anlässe auBer dem Kommentar ein positives Vorzeichen aufweisen.

Tabelle 1: OLS-Regressionsanalyse zu Determinanten der Valenz von Zeitungsartikeln zum Elterngeld 2004-2012

\begin{tabular}{|c|c|c|c|c|c|c|c|c|}
\hline \multirow[b]{2}{*}{ Jahr } & \multicolumn{2}{|c|}{ Modell 1} & \multicolumn{2}{|c|}{ Modell 2} & \multicolumn{2}{|c|}{ Modell 3} & \multicolumn{2}{|c|}{ Modell 4} \\
\hline & $\begin{array}{l}-0,003 \\
(0,031)\end{array}$ & $-0,009$ & $\begin{array}{c}0,002 \\
(0,029)\end{array}$ & 0,007 & & & & \\
\hline Summe der Ziele & $\begin{array}{l}0,283^{* *} \\
(0,098)\end{array}$ & 0,446 & $\begin{array}{l}0,286^{* *} \\
(0,061)\end{array}$ & 0,451 & $\begin{array}{c}0,248^{* *} \\
(0,092)\end{array}$ & 0,391 & $\begin{array}{l}0,273^{\star *} \\
(0,061)\end{array}$ & 0,430 \\
\hline Anlass Medien- Kommentar & $\begin{array}{l}-0,486^{*} \\
(0,202)\end{array}$ & $-0,249$ & $\begin{array}{l}-0,155 \\
(0,343)\end{array}$ & $-0,079$ & $\begin{array}{l}-0,480^{*} \\
(0,192)\end{array}$ & $-0,246$ & $\begin{array}{l}-0,551^{* *} \\
(0,190)\end{array}$ & $-0,282$ \\
\hline Anlass Studie (Wissenschaft) & $\begin{array}{l}0,538^{*} \\
0,269\end{array}$ & 0,206 & $\begin{array}{c}0,801^{*} \\
(0,381)\end{array}$ & 0,307 & $\begin{array}{c}0,536^{*} \\
(0,263)\end{array}$ & 0,205 & $\begin{array}{c}0,403 \\
(0,252)\end{array}$ & 0,155 \\
\hline Anlass amtliche Statistik (StBA) & $\begin{array}{c}0,745 \\
(0,484)\end{array}$ & 0,148 & $\begin{array}{c}1,070 \\
(0,561)\end{array}$ & 0,213 & & & & \\
\hline Anlass Gesetz/Information & $\begin{array}{c}0,488 \\
(0,684)\end{array}$ & 0,069 & $\begin{array}{c}1,144 \\
(0,727)\end{array}$ & 0,162 & & & & \\
\hline Anlass Politik & & & $\begin{array}{c}0,404 \\
(0,311)\end{array}$ & 0,255 & & & & \\
\hline Anlass Verband & $\begin{array}{l}-0,390 \\
(0,311)\end{array}$ & $-0,120$ & & & & & & \\
\hline Ziel Geburtenrate erhöhen & $\begin{array}{l}-0,144 \\
(0,222)\end{array}$ & $-0,094$ & & & $\begin{array}{l}-0,095 \\
(0,209)\end{array}$ & $-0,062$ & & \\
\hline Ziel Zeit bzw. Schonraum & $\begin{array}{c}0,258 \\
(0,252) \\
\end{array}$ & 0,135 & & & $\begin{array}{c}0,314 \\
(0,233) \\
\end{array}$ & 0,165 & & \\
\hline Konstante & 7,095 & $(63,078)$ & $-2,768$ & $(58,795)$ & 1,708 & $(0,129)^{\star *}$ & 1,710 & $(0,129)$ \\
\hline $\mathrm{N}$ & & 83 & & 33 & & 33 & & 3 \\
\hline $\mathrm{R}^{2}$ & & $355^{\star *}$ & 0,3 & $29^{* *}$ & & $13^{* *}$ & $0,2 \varepsilon$ & $84^{* *}$ \\
\hline $\mathrm{R}_{\text {korr }}^{2}$ & & $275^{\star *}$ & & $66^{\star *}$ & & $69^{* *}$ & 0,25 & $56^{* *}$ \\
\hline
\end{tabular}

Anmerkungen: Für jede unabhängige Variable steht in der linken Spalte eines Modells der nicht standardisierte partielle Regressionskoeffizient b, darunter in Klammern der Standardfehler. In der rechten Spalte ist der standardisierte partielle Regressionskoeffizient Beta zu finden. * bedeutet Signifikanzniveau > $95 \%, * *>99 \%$. 


\section{Zusammenfassung und Diskussion}

Der Beitrag hat die jeweiligen charakteristischen Merkmale der Diskurse in den Sphären Politik, Medien und Wissenschaft gezeigt und durch welche Mechanismen diese wechselseitig verknüpft sind. Die Analyse der Ziele im Vergleich zwischen parlamentarischen Dokumenten und Medienanalyse sowie die Valenzanalyse der Zeitungsartikel von 2004 bis 2012 haben verdeutlicht, dass die Definition von Zielen des Elterngelds der Schlüssel zum Verständnis der Diskurse und der Bewertung des BEEG in der Öffentlichkeit ist.

Die Interaktion zwischen Politik, Wissenschaft und Medien ist für die Entstehung des BEEG aufschlussreich. Henninger und Wahl (2010: 363) haben zu Recht auf die Akteurskomponente hingewiesen, wonach „die sich veränderte politische Interessenkonstellation durch strategisches Handeln zunutze“ gemacht wurde, ,um so Widerstände gegen Reformen zu überwinden“. Darüber hinaus hat der Siebte Familienbericht eine Begründung für das Elterngeld geliefert, die für verschiedene Interessengruppen und Parteiflügel beider Volksparteien attraktiv war. Der Willenbildungsprozess seit der Kabinettsklausur am 5.9.2004 und die Kommissionsarbeit des Siebten Familienberichts waren zeitlich und inhaltlich eng vertaktet. Insbesondere bei den beiden inhaltlichen Hürden im Willensbildungsprozess, der Verteilungsfrage und den Vätermonaten, war das Zusammenwirken von Regierungsarbeit und den Argumenten der Wissenschaft essentiell.

Für die Medien war und ist das Elterngeld ein viel beachtetes Thema, dessen Kräftefeld hier durch die Kombination aus Frequenz- und Valenzanalyse für den Zeitraum 2004 bis 2012 beleuchtet wurde. Bei der Berichterstattung in den Medien standen demografische Wirkungen des Elterngelds bzw. ihr Ausbleiben und Diskussionen von politischen Akteuren um Bewertung, Änderungen oder gar Abschaffung des Elterngelds im Mittelpunkt.

Die Ziele der Elterngelds - sowohl in den Medien als auch in der Politik - lassen sich auf fünf Dimensionen verdichten: Einkommen sichern, Zeit und Schonraum, steigende Erwerbsbeteiligung (von Müttern), Gleichstellung und Geburtenrate erhöhen. Die Analysen zeigen jedoch, dass sich die Zielhierarchie erheblich unterscheidet (siehe Abb. 5): Während die Erhöhung der Geburtenrate das zentrale Ziel in den Medien darstellt, rangiert es in den parlamentarischen Dokumenten ganz hinten. Umgekehrt spielen Zeit und Schonraum für Familien bei den politischen Zielen eine zentrale Rolle, während dies in den Medien weniger erwähnt wird.

Ein solcher Bias der Zielhierarchien zwischen Gesetzgeber und Medien kann zu fatalen Konsequenzen führen. Hier kann man aus dieser Analyse zum Elterngeld grundsätzliche Erkenntnisse extrahieren: Wenn ein für den Gesetzgeber eher nachrangiges Ziel nicht erreicht wird, die prioritären Ziele einer Maßnahme jedoch adäquat erreicht werden, kann man prinzipiell von einem erfolgreichen Gesetz sprechen. Wenn allerdings in den Medien und damit in der Öffentlichkeit ein solches nachrangiges Ziel in den Mittelpunkt gestellt wird und die anderen Effekte in den Hintergrund treten, kann dies die Akzeptanz einer Maßnahme beschädigen. Für Öffentlichkeitsarbeit und Evaluation von Politikmaßnahmen ist dies hochgradig relevant, zumal bei einem besonderen Bedarf an Outcome-Legitimation: Die wissenschaftliche Analyse von Wirkungen ist nur eine Voraussetzung für Bewertungen, die normative Benennung der Ziele und ihrer Hierarchie ist die zweite. Um eine verzerrte Wiedergabe von Zielen in den Medien zu vermeiden, ist eine klare, strategische und konsistente Kommunikation der Ziele von Seiten der Politik notwendig. Der 
ELTERN-Studie (2013) nach haben 61\% der Befragten nicht den „Eindruck, dass die derzeitige Bundesregierung konkrete familienpolitische Ziele verfolgt". Auch dies verdeutlicht, dass die Kommunikation der Ziele der Familienpolitik die Adressaten kaum erreicht hat. Widersprüchliche Äußerungen von Politikern oder die Fokussierung auf singuläre Ziele können zu einer verzerrten und ggf. negativen Berichterstattung in den Medien führen.

Abbildung 5: Unterschiede der Zielhierarchie des Elterngelds in Medien und Politik

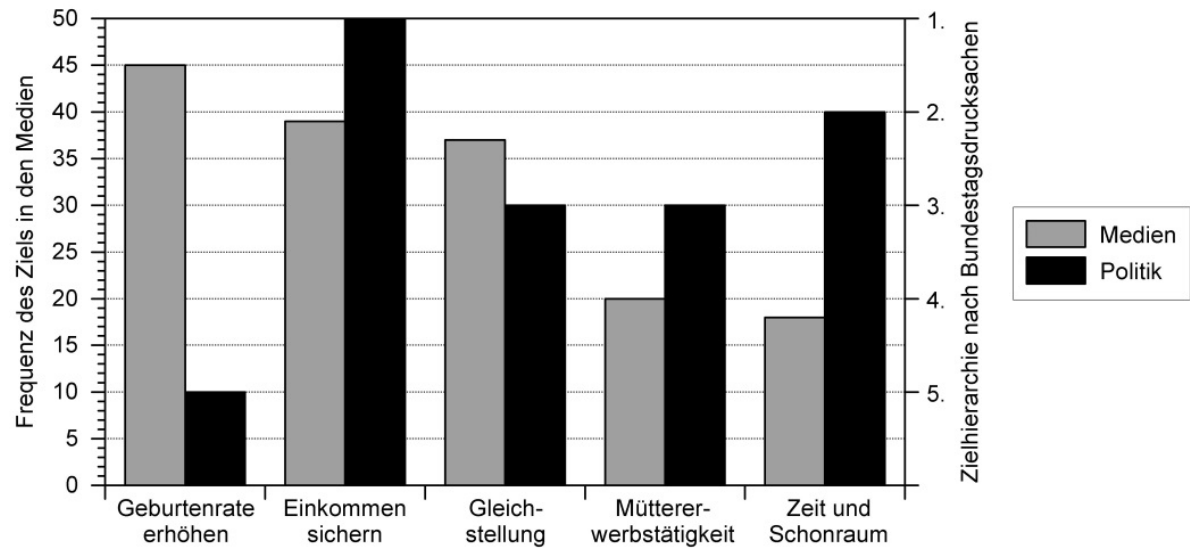

Anmerkungen: Die Werte für Medien beziehen sich auf die Frequenz der jeweiligen Zielnennungen zwischen 2004-2012. Die Rangfolge für den Bereich Politik basiert auf der Inhaltsanalyse der Bundestagsdrucksachen im parlamentarischen Entstehungsprozess des BEEG (Deutscher Bundestag 2006b, 2006c, 2006d, 2006e, 2006f).

Die Valenzanalyse unterstreicht die Bedeutung der Ziele für die mediale Berichterstattung - und damit auch für den öffentlichen Diskurs, da die Medien die Wahrnehmung der Bürger und den öffentlichen Diskurs maßgeblich prägen. Dabei zeigt sich, dass weniger die Auswahl eines bestimmten Ziels entscheidend ist, sondern vielmehr die Summe der Ziele sich als hochsignifikant erweist. Je mehr Ziele mit dem Elterngeld verbunden werden, desto positiver ist die Bewertung. Die Schlussfolgerungen für die Politikberatung liegen auf der Hand: Es ist weniger hilfreich, ein Ziel, dessen Wirkung gerade Konjunktur hat, zu betonen. Insbesondere wenn dessen Wirkungsbefund nicht kausal ist und zu einem späteren Zeitpunkt anders aussehen könnte - so geschehen bei dem im September 2008 verkündeten vermeintlichen Anstieg der Geburtenrate. Vielmehr könnte eine Kommunikationsstrategie zu einer positiven Valenz führen, die konsequent den Fünfklang der Ziele betont. Die Valenzanalyse verdeutlicht, wie zentral eine klare und explizite Zieldefinition für die familienpolitische Kommunikation ist (vgl. Schneider et al. 2013).

Die Analyse hat zudem gezeigt, dass die Valenz auch von den Anlässen modifiziert wird. Journalistische Leitkommentare sind mit einer tendenziell negativen Valenz assoziiert, wissenschaftliche Befunde mit einer positiven. Eine Interpretation im Sinne des Medienstereotyps „only bad news are good news“ wäre zu einfach, denn die statistischen Tendenzen verdecken die enorme Vielfältigkeit der Meinungen und Argumente, die die Medienanalyse zu Tage gebracht hat. Während die positive Valenz mit der Berücksichti- 
gung möglichst mehrerer Ziele verbunden ist, beruhen negative Valenzen v.a. auf drei Argumenten: Haushaltskosten von rund $5 \mathrm{Mrd}$. $€$, Verteilungsfragen und die nicht gestiegene Geburtenrate. Das Kostenargument verkennt dabei oft, dass die Vorgängermaßnahme Erziehungsgeld ein Volumen von 2,8 Mrd. $€$ hatte und das Elterngeld nur 2,5\% der familienpolitischen Ausgaben nach der breiten Definition (BMFSFJ 2012) einnimmt.

Die Kritik einer kurzfristig nicht gestiegenen Geburtenrate ist fragwürdig, da die Wirkmechanismen sich nur langfristig und in Interaktion mit anderen Maßnahmen entfalten (Bujard 2011). Allerdings ist der häufige mediale Verweis auf dieses Ziel insofern nachvollziehbar, da es im Entstehungsprozess des BEEG ein valentes Ziel war, was nicht zuletzt die Zitate von Bundeskanzler Schröder sowie den Familienministerinnen Schmidt und von der Leyen, aber auch von CDU/CSU-Fraktionschef Volker Kauder belegen. Aufgrund der genannten Wirkmechanismen auf die Geburtenentwicklung und der Kommunikation der vier anderen Ziele als prioritär, wäre eine Delegitimierung des Elterngelds auf Basis dieses Kritikpunkts unplausibel. Die offene Flanke des Elterngelds liegt eher bei der Verteilungswirkung. So nachvollziehbar die lebenslaufbezogene Argumentation ist, bleibt es eine Herausforderung, die höheren Leistungen für Mittelschichtfamilien, akademische Berufsfelder und ältere Eltern gegenüber anderen, weniger privilegierten Gruppen zu kommunizieren. Dass dieser potenzielle Kritikpunkt in den letzten Jahren relativ zurückgegangen ist, mag erstaunlich sein; eine Erklärung wäre mit Bezug auf die Parteipolitik die, dass bei Christdemokraten eher Geburtenentwicklung, Müttererwerbstätigkeit und Betreuungsgeld im Fokus standen und in der SPD die Gleichstellungsaspekte und die Modernisierung der Familienpolitik den Diskurs dominiert haben. Die Verteilungsfrage ist allerdings ein wichtiger Aspekt für eine mögliche weitere Reform des Elterngelds.

Letztlich hat das Elterngeld den Vorteil, dass es mehreren als sinnvoll erachteten Zielen gleichzeitig dienen kann. Diese komplementäre Zielstruktur ist für die politische Kommunikation eine große Chance. Dies hat aber auch Konsequenzen für die Wissenschaft: Die fünf Zieldimensionen, die sich sowohl in der politischen Arena als auch in der medialen Berichterstattung identifizieren lassen, sind ein wichtiger Orientierungspunkt für Wirkungsanalysen. Denn ob intendiert oder nicht: Wissenschaftliche Analysen zum Elterngeld können enorme poltische und mediale Wellen auslösen. Dabei kann die Auswahl des analysierten Ziels ausschlaggebend sein - sie sollte daher in den Kontext des Zielkanons gestellt werden.

\section{Danksagung}

Der Autor dankt Jasmin Passet, Norbert F. Schneider, Stefan Wurster, Thomas Zerback und zwei anonymen Gutachterinnen und Gutachtern für wertvolle Hinweise, Evelyn Grünheid für Korrektorat sowie Sybille Steinmetz, Christian Fiedler und Bernhard Gückel für Beratung bei Grafiken und der Medienanalyse. 


\section{Literatur}

Bannas, G. (2004). Schröder: Der Umbau des Sozialstaates wird fortgesetzt. Klausurtagung in Bonn / Elterngeld statt Erziehungsgeld. In: $F A Z$ vom 5.9.2004.

Bertram, H., Krüger, H. \& Spieß, C. K. (2006). Wem gehört die Familie der Zukunft? Expertisen zum 7. Familienbericht der Bundesregierung. Opladen: Barbara Budrich.

Bertram, H., Rösler, W. \& Ehlert, N. (2005). Nachhaltige Familienpolitik. Zukunftssicherung durch einen Dreiklang von Zeitpolitik, finanzieller Transferpolitik und Infrastruktur. Gutachten für das BMFSFJ: Berlin: Bundesministerium für Familie, Senioren, Frauen und Jugend.

Bundesministerium für Familie, Senioren, Frauen und Jugend (BMFSFJ) (2012). Bestandsaufnahme der familienbezogenen Leistungen und Maßnahmen des Staates im Jahr 2010. In: http://www. bmfsfj.de/BMFSFJ/familie,did=158318.html (Stand: 2013-02-06).

Bujard, M. (2011). Geburtenrückgang und Familienpolitik. Baden-Baden: Nomos.

Deutscher Bundestag (2006a). Siebter Familienbericht. Familie zwischen Flexibilität und Verlässlichkeit. Perspektiven für eine lebenslaufbezogene Familienpolitik. Berlin: Deutscher Bundestag (Drucksache 16/1360).

Deutscher Bundestag (2006b). Gesetzentwurf der Fraktionen der CDU/CSU und SPD zur Einführung des Elterngeldes. Berlin: Deutscher Bundestag (Drucksache 16-1889).

Deutscher Bundestag (2006c). Gesetzentwurf der Bundesregierung zur Einführung des Elterngeldes. Berlin: Deutscher Bundestag (Drucksache 16-2454).

Deutscher Bundestag (2006d). Beschlussempfehlung und Bericht des Ausschusses für Familie, Senioren, Frauen und Jugend. Berlin: Deutscher Bundestag (Drucksache 16-2785).

Deutscher Bundestag (2006e). Bericht des Haushaltsausschusses. Berlin: Deutscher Bundestag (Drucksache 16-2788).

Deutscher Bundestag (2006f). Entschließungsantrag der Fraktion der FDP. Berlin: Deutscher Bundestag (Drucksache 16-2809).

Easton, D. (1965). A framework for political analysis. Englewood Cliffs: Prentice-Hall.

ELTERN (Hrsg) (2013). ELTERN-Studie „Wenn Eltern die Wahl haben“. In: http://eltern.de/c/pdf/ ELTERN forsa-Studie Wahl.pdf

Gerlach, I. (2004). Familienpolitik. Wiesbaden: VS Verlag für Sozialwissenschaften.

Gruescu, S. \& Rürup, B. (2005). Nachhaltige Familienpolitik. Essay. Aus Politik und Zeitgeschichte, 2324/2005, S. 3-6.

Henninger, A. \& Wahl, A. (2010). Das Umspielen von Veto-Spielern. Wie eine konservative Familienministerin den Familialismus des deutschen Wohlfahrtsstaates unterminiert. In: Egle, C. \& Zohlnhöfer, R. (Hrsg.), Die zweite Große Koalition. Eine Bilanz der Regierung Merkel 2005-2009. Wiesbaden: VS Verlag für Sozialwissenschaften, S. 361-379.

Jann, W. \& Wegrich, K. (2003). Phasenmodelle und Politikprozesse: Der Policy Cycle. In: Schubert; K. \& Bandelow, N. C. (Hrsg.), Lehrbuch der Politikfeldanalyse. München: Oldenbourg, S. 71105 .

Jun, U. \& Grabow, K. (2008). Mehr Expertise in der deutschen Politik? Gütersloh: Bertelsmann Stiftung.

Kamann, M. (2004). Eltern. Kommentar. In: Die Welt vom 6.9.2004, S. 3.

Kauder, V. (2012). Interview. In: Süddeutsche Zeitung vom 6.7.2012.

Kaufmann, F.-X. (2002). Politics and policies towards the family in Europe: A framework and an inquiry into their differences and convergences. In: Kaufmann, F.-X. et al., Family life and family policies in Europe, Oxford: University Press, S. 419-490.

Kluve, J. \& Tamm, M. (2009). Now daddy's changing diapers and mommy's making her career. Evaluating a generous parental leave regulation using a natural experiment. Bonn: Forschungsinstitut zur Zukunft der Arbeit (IZA) (IZA Discussion Paper No. 4500).

Krippendorff, K. (2004). Content analysis: An introduction to its methodology. Thousand Oaks, CA: Sage. 
Ristau, M. (2005). Der ökonomische Charme der Familie. Aus Politik und Zeitgeschichte, 23-24/2005, S. $16-23$.

Rürup, B. \& Gruescu, S. (2003). Nachhaltige Familienpolitik im Interesse einer aktiven Bevölkerungsentwicklung. Gutachten für das BMFSFJ. Berlin: Bundesministerium für Familie, Senioren, Frauen und Jugend.

Rürup, B. \& Schmidt, R. (2003). Nachhaltige Familienpolitik im Interesse einer aktiven Bevölkerungspolitik. In: www.bmfsfj.de/Kategorien/aktuelles/did=12334.html (Stand: 2007-07-15).

Schmidt, R. (2012). „Wie das Ungeheuer von Loch Ness“. In: $F A Z$ vom 15.7.2012, S. 9.

Schneider, N. F., Bujard, M., Henry-Huthmacher, C., Mayer, T., Possinger, J. \& Speich, M. (2013). Moderne Familien - neue Politik: Ziele, Strategien und Handlungsebenen einer nachhaltigen Familienpolitik. Impulspapier zur Familienpolitik im Rahmen der Demografiestrategie der Bundesregierung, Berlin: BMFSFJ. http://www.bmfsfj.de/BMFSFJ/Familie/demografischer-wandel,did=190036. html.

Schnell, R., Hill, P. \& Esser, E. (2005). Methoden der empirischen Sozialforschung. München: Oldenbourg (7. Auflage).

Von der Leyen, U. (2008). „Ein uneingeschränkter Erfolg““. In: FAZ.NET vom 30.10.2008.

Wagschal, Uwe (1999). Statistik für Politikwissenschaftler. München: Oldenbourg.

Wingen, M. (1997). Familienpolitik: Grundlagen und aktuelle Probleme. Stuttgart: Lucius und Lucius.

Wolber, C. (2006). Elterngeld - Rückendeckung für Ministerin von der Leyen. In: Berliner Morgenpost vom 20.5.2006. http://www.morgenpost.de/printarchiv/politik/article269976

Wrohlich, K., Berger, E., Geyer, J., Haan, P., Sengül, D., Spieß, C. K. \& Thiemann, A. (2012). Elterngeldmonitor. Berlin: Bundesministerium für Familie, Senioren, Frauen und Jugend (BMFSFJ) und Deutsches Institut für Wirtschaftsforschung (DIW).

Eingereicht am/Submitted on: 26.04.2013

Angenommen am/Accepted on: 17.07.2013

Anschrift des Autors/Address of the author:

Dr. Martin Bujard

Bundesinstitut für Bevölkerungsforschung (BiB)

Friedrich-Ebert-Allee 4

65185 Wiesbaden

E-Mail:martin.bujard@bib.bund.de 


\section{Anhang}

Tabelle 2: OLS-Regressionsanalyse zu Determinanten der Valenz von Zeitungsartikeln zum Elterngeld 2004-2012 (Gesamtsample inklusive Artikel mit neutraler Valenz)

\begin{tabular}{|c|c|c|c|c|c|c|c|c|}
\hline \multirow[b]{2}{*}{ Jahr } & \multicolumn{2}{|c|}{ Modell 1b } & \multicolumn{2}{|c|}{ Modell 2b } & \multicolumn{2}{|c|}{ Modell 3b } & \multicolumn{2}{|c|}{ Modell 4b } \\
\hline & $\begin{array}{l}-0,008 \\
(0,016)\end{array}$ & $-0,034$ & $\begin{array}{l}-0,003 \\
(0,016)\end{array}$ & $-0,013$ & & & & \\
\hline Summe der Ziele & $\begin{array}{c}0,184^{* *} \\
(0,057)\end{array}$ & 0,402 & $\begin{array}{c}0,211^{* *} \\
(0,033)\end{array}$ & 0,460 & $\begin{array}{c}0,171^{\text {** }} \\
(0,056)\end{array}$ & 0,373 & $\begin{array}{c}0,195^{* \star} \\
(0,032)\end{array}$ & 0,425 \\
\hline Anlass Medien- Kommentar & $\begin{array}{l}-0,614 \\
(0,351)\end{array}$ & $-0,359$ & $\begin{array}{l}-0,275 \\
(0,201)\end{array}$ & $-0,161$ & $\begin{array}{l}-0,486^{* *} \\
(0,119)\end{array}$ & $-0,284$ & $\begin{array}{l}-0,518^{* \star} \\
(0,120)\end{array}$ & $-0,303$ \\
\hline Anlass Studie (Wissenschaft) & $\begin{array}{c}0,122 \\
(0,349)\end{array}$ & 0,070 & $\begin{array}{c}0,433^{*} \\
(0,202)\end{array}$ & 0,247 & $\begin{array}{c}0,233 \\
(0,120)\end{array}$ & 0,133 & $\begin{array}{c}0,177 \\
(0,119)\end{array}$ & 0,101 \\
\hline Anlass amtliche Statistik (StBA) & $\begin{array}{c}0,004 \\
(0,351)\end{array}$ & 0,002 & $\begin{array}{c}0,348 \\
(0,210)\end{array}$ & 0,183 & & & & \\
\hline Anlass Gesetz/Information & $\begin{array}{l}-0,008 \\
(0,340)\end{array}$ & $-0,005$ & $\begin{array}{c}0,357 \\
(0,192)\end{array}$ & 0,253 & & & & \\
\hline Anlass Politik & $\begin{array}{l}-0,147 \\
(0,335)\end{array}$ & $-0,138$ & $\begin{array}{c}0,229 \\
(0,175)\end{array}$ & 0,216 & & & & \\
\hline Anlass Verband & $\begin{array}{l}-0,485 \\
(0,383)\end{array}$ & $-0,166$ & & & & & & \\
\hline Ziel Geburtenrate erhöhen & $\begin{array}{l}-0,050 \\
(0,130)\end{array}$ & $-0,041$ & & & $\begin{array}{l}-0,071 \\
(0,128)\end{array}$ & $-0,059$ & & \\
\hline Ziel Zeit bzw. Schonraum & $\begin{array}{c}0,330 \\
(0,155)\end{array}$ & 0,188 & & & $\begin{array}{c}0,309^{*} \\
(0,151)\end{array}$ & 0,176 & & \\
\hline Konstante & 17,786 & $(32,789)$ & 7,475 & $(32,649)$ & $1,905(C$ & $0,047)^{\star *}$ & 1,906 & $(0,047)^{* *}$ \\
\hline $\mathrm{N}$ & & 176 & & 176 & & 76 & & 76 \\
\hline $\mathrm{R}^{2}$ & & $281^{* *}$ & & $243^{* *}$ & & $54^{* *}$ & & $24^{* *}$ \\
\hline $\mathrm{R}_{\text {korr }}^{2}$ & & $237^{* *}$ & & $212^{* *}$ & & $32^{* *}$ & & $11^{\text {** }}$ \\
\hline
\end{tabular}

Anmerkungen: Diese Regressionsanalyse umfasst alle 176 Zeitungsartikel, im Unterschied zu Tabelle 1 also auch jene, die eine neutrale Valenz aufweisen. Hier wurde die Valenz in drei Abstufungen kodiert: positiv (3), neutral oder ambivalent (je 2) und negativ (1). Die Befunde bestätigen weitestgehend die in Tabelle 1, die trotz der geringeren Fallzahl aus inhaltlichen und methodischen Erwägungen vorgezogen ist. Erläuterungen siehe Tabelle 1 und Text. 